\section{O uso da fototerapia em recém-nascidos: avaliação da prática clínica}

\section{Phototherapy in the newborn infant: evaluation of clinical practice}

\author{
Alan Araújo Vieira 1 \\ Carmem Lúcia Mendonça Accetta Lima 2 \\ Manoel de Carvalho 3 \\ Maria Elisabeth Lopes Moreira 4 \\ 1,3,4 Instituto Fernandes Figueira. Avenida Rui Barbosa, 716 \\ Flamengo. Rio de Janeiro, RJ, Brasil. CEP: 22.250-020. \\ Tel: 2125530052 \\ 2 Universidade do Grande Rio. Duque de Caxias, RJ, Brasil.
}

\begin{abstract}
Objectives: to describe the use of phototherapy in the day to day clinical practice by healthcare professionals of public maternity hospitals in the city of Rio de Janeiro.

Methods: heads of healthcare departments, doctors and nurses of 17 public maternity hospitals were interviewed on issues related to phototherapy management in their services.

Results: eighty nine healthcare professionals were interviewed. Seventy four per cent of the doctors noted there was a written guideline for neonatal jaundice management in their departments but with a great variation of responses related to the medical conduct adopted, including among professionals working in the same unit, $74 \%$ of doctors prescribed prophylactic phototherapy and $64 \%$ said they increased fluid intake during treatment. Distance placed between the newborn and the irradiance source varied from 20 to $70 \mathrm{cms}$. Half of the respondents noted the lack of guidelines to verify irradiation during phototherapy. A marked variation of bilirubin serum levels were used for phototherapy and exchange blood transfusion indications.

Conclusions: our findings suggest the lack of consensus among healthcare professionals related to the use of phototherapy and that some of the routinely adopted practices could impair treatment efficacy.

Key words Jaundice, Infant newborn, Phototherapy, Hyperbilirubinemia
\end{abstract}

\section{Resumo}

Objetivos: descrever o uso da fototerapia na prática clínica diária pelos profissionais de saúde das maternidades públicas da cidade do Rio de Janeiro.

Métodos: chefes de serviço, médicos e profissionais da área de enfermagem de 17 maternidades públicas foram entrevistados sobre questões operacionais relacionadas ao manuseio das fototerapias em seus serviços.

Resultados: oitenta e nove profissionais de saúde foram entrevistados. Setenta e quatro por cento dos médicos afirmaram a existência de uma rotina escrita para o tratamento da icterícia neonatal em seu serviço, havendo, porém, grande variabilidade nas respostas quanto às condutas adotadas, inclusive entre profissionais de uma mesma unidade; $74 \%$ dos médicos prescrevem fototerapia profilática e $64 \%$ afirmaram aumentar a taxa hídrica durante o tratamento. A distância utilizada entre o recém-nascido e a fonte luminosa variou de 20 a $70 \mathrm{~cm}$. Metade dos entrevistados afirmou que não havia uma rotina para a verificação da irradiância durante a fototerapia. Observou-se enorme variação nos níveis séricos de bilirrubina utilizados para a indicação de fototerapia e exanguineotransfusão.

Conclusões: os resultados sugerem que não existe um consenso entre os profissionais de saúde quanto ao uso da fototerapia, sendo que algumas práticas adotadas rotineiramente podem diminuir a eficácia do tratamento.

Palavras-chave Icterícia, Recém-nascido, Fototerapia, Hiperbilirrubinemia. 


\section{Introdução}

A icterícia é uma patologia muito comum no período neonatal. Cerca de $60 \%$ dos recém-nascidos desenvolvem hiperbilirrubinemia clinicamente detectável nos primeiros dias de vida. Na maioria das vezes em que ocorre, representa um fenômeno fisiológico transitório, não requerendo intervenção terapêutica. ${ }^{1}$ A fototerapia é, sem dúvida, a modalidade terapêutica mais utilizada mundialmente para o tratamento da icterícia neonatal. ${ }^{2}$ Estima-se que nos Estados Unidos um número superior a 350.000 recém-nascidos recebam, anualmente, esse tratamento. ${ }^{3}$ Entretanto, apesar da vasta literatura médica a respeito de seu mecanismo de ação, efeitos biológicos e complicações, existe, ainda, considerável desinformação acerca do uso clínico da fototerapia por profissionais de saúde.

Desde a descoberta da fototerapia há 40 anos, não só as indicações para seu uso mudaram consideravelmente como novos e mais eficazes modelos foram introduzidos no mercado.

Em 1994, a Academia Americana de Pediatria (AAP) desenvolveu critérios para a abordagem clínica do recém-nascido ictérico, visando otimizar o uso da fototerapia. 4 A não observância de critérios técnicos adequados para o uso dessa tecnologia pode prejudicar a eficácia terapêutica e a qualidade do tratamento oferecido ao recém-nascido (RN) ictérico.

O objetivo deste estudo é descrever como os profissionais de saúde das maternidades públicas da cidade do Rio de Janeiro utilizam a fototerapia na sua prática clínica diária e discutir as possíveis implicações dessas condutas na eficácia terapêutica dessa tecnologia.

\section{Métodos}

Trabalho descritivo embasado em dados coletados por meio da aplicação de um questionário com perguntas abertas e fechadas, segundo modelo utilizado por Hansen, realizado de junho a dezembro de 2001.5

Foram estudados todos os hospitais localizados no Município do Rio de Janeiro que possuíam, à época do estudo, condições básicas para oferecerem tratamento à icterícia neonatal. Ao todo, foram selecionados 17 hospitais, sendo 13 municipais e quatro universitários.

Os questionários foram aplicados pelo investigador durante entrevista direta com os chefes de serviço das unidades, com os médicos neonatologistas (pelo menos três em cada serviço) e profissionais da área de enfermagem (enfermeiros e técnicos - pe- lo menos dois em cada serviço) que estavam de plantão no dia da visita do pesquisador e tinham disponibilidade, naquele momento, para responder ao questionário proposto.

Foi ressaltado, durante as entrevistas, que a intenção da pesquisa era descrever a prática clínica diária, não importando os conhecimentos teóricos sobre os itens abordados.

O questionário continha perguntas sobre quatro pontos específicos:

- Tipos de tratamentos disponíveis para a icterícia neonatal (fototerapia, exanguineotranfusão, drogas).

- Tipos de aparelhos de fototerapias disponíveis no serviço (convencional, spot, colchão de fibra ótica ou Biliberço®).

- Conhecimentos técnicos sobre os aparelhos de fototerapia - a quantidade e os tipos de lâmpadas existentes em cada aparelho (lâmpadas brancas, azuis ou halógenas), a frequiência de aferição da irradiância emitida $(\mu \mathrm{W} / \mathrm{cm} 2)$ durante a fototerapia e a frequiência da troca das lâmpadas dos aparelhos.

- Existência de rotina escrita que determinasse, entre outras, a distância entre o RN e o foco luminoso, a necessidade de aumento de taxa hídrica durante o tratamento fototerápico, o uso de proteção para os olhos e as gônadas, o uso de fraldas durante o tratamento, a manutenção de aleitamento materno durante o tratamento, o uso de fototerapia profilática ou dupla, o posicionamento dos focos luminosos no corpo do RN, a freqüência de aferição da bilirrubina sérica para controle do tratamento e os níveis séricos indicativos de fototerapia e exanguineotransfusão (EXT) em diferentes faixas de peso ao nascimento.

Dadas as características envolvidas, algumas perguntas foram direcionadas apenas aos médicos, pois alguns cuidados de recém-nascidos ictéricos em fototerapia são inerentes, apenas, à decisão médica.

Cinqüienta médicos, 30 enfermeiros e nove técnicos de enfermagem de 17 maternidades da rede pública da cidade do Rio de Janeiro foram entrevistados.

Este trabalho foi aprovado pelo comitê de Ética em Pesquisa do Instituto Fernandes Figueira (IFF) e dos Hospitais onde foram realizadas as entrevistas.

\section{Resultados}

Foram entrevistados 89 profissionais de saúde, sendo 50 médicos (13 chefes de serviço, 3 médicos residentes e 34 médicos neonatologistas plantonistas) e 39 profissionais de enfermagem (30 enfermeiras e 9 técnicos de enfermagem). Apenas três 
questionários foram respondidos por médicos residentes, todos de uma mesma unidade hospitalar; nas demais maternidades, não foram encontrados médicos residentes no momento da visita do pesquisador. Os resultados a seguir estão apresentados em bloco, exceto quando provenientes de perguntas direcionadas apenas a uma das categorias profissionais, pois consideramos que isso representa melhor o objetivo do estudo, que é descrever como a fototerapia é utilizada na prática clínica diária.

\section{Existência de rotina escrita}

\section{sobre o tratamento do RN ictérico}

Dos 50 médicos questionados, $74 \%$ afirmaram que havia uma "rotina" escrita sobre o uso da fototerapia em seu serviço.

\section{Modalidades de tratamento disponíveis}

Dentre as modalidades terapêuticas disponíveis para o tratamento da icterícia neonatal, a fototerapia estava presente em todas as unidades estudadas. A EXT foi relatada como forma de tratamento por $94 \%$ dos médicos entrevistados e o tratamento farmacológico foi relatado por $72 \%$, sendo que o fenobarbital foi a droga de escolha e a colestase neonatal sua principal indicação. $\mathrm{O}$ uso da imunoglobulina endovenosa foi relatado por $10 \%$ dos médicos em caso de incompatibilidade sanguínea Rh grave.

\section{Tipos de aparelhos de fototerapia utilizados}

Todas as unidades pesquisadas possuíam aparelhos de fototerapia tipo spot halógeno (Bilispot $\left.{ }^{\circledR}\right)$. Aparelhos convencionais de fototerapia estavam presentes em 14 unidades e aparelhos de fototerapia de contato em quatro unidades (Biliberço® em três e Biliblanket ${ }^{\circledR}$ em uma unidade).

As 14 unidades que possuíam aparelhos convencionais de fototerapia utilizavam luz fluorescente brancas (tipo luz do dia), sendo que oito unidades utilizavam também aparelhos de fototerapia equipados com uma mistura de lâmpadas fluorescentes brancas e azuis. Três unidades utilizavam aparelhos de fototerapia equipados exclusivamente com lâmpadas fluorescentes azuis. Os aparelhos de fototerapia eram equipados com seis a oito lâmpadas fluorescentes por aparelho. (Tabela 1)

\section{Verificação da irradiância}

Quanto à verificação da irradiância, 50\% dos profissionais entrevistados afirmaram que existia uma rotina no serviço para tal fim, $44 \%$ afirmaram que não existia e $6 \%$ não souberam informar. Houve discrepâncias entre as respostas em uma mesma unidade.

Dos 45 profissionais que afirmaram existir uma rotina de verificação da irradiância, 21 não souberam informar a frequiência em que ocorria e os demais relataram desde avaliação diária até a cada 60 dias. (Tabela 1) A maioria desses profissionais (21) não soube informar de que maneira ou com que instrumento a irradiância era medida.

\section{Troca das lâmpadas \\ dos aparelhos de fototerapia}

Trinta e quatro por cento dos profissionais entrevistados declararam que as lâmpadas fluorescentes eram trocadas regularmente, $37 \%$ afirmaram que as lâmpadas nunca eram trocadas e $29 \%$ não souberam responder. Dos 30 profissionais que afirmaram que as lâmpadas eram trocadas regularmente, 26 não souberam precisar a frequiência das trocas. Dos quatro restantes, um relatou troca a cada quatro dias, um a cada 7 dias e dois a cada 90 dias.

\section{Distância entre a fonte luminosa e o RN}

Quando questionados a respeito de uma distância padronizada entre o recém-nascido ictérico e a fonte luminosa, $96 \%$ dos profissionais afirmaram que ela existia em seu serviço, porém, as respostas variaram muito, inclusive entre profissionais de um mesmo hospital (de $20 \mathrm{~cm}$ até $70 \mathrm{~cm}$ ). (Tabela 1)

\section{Manuseio hídrico do RN em fototerapia}

A conduta em relação à oferta hídrica para o recémnascido ictérico durante a fototerapia também se mostrou variável: 32 (64\%) dos médicos afirmaram prescrever, rotineiramente, aumento da oferta de líquidos. Observamos, entretanto, grande variabilidade nas respostas, inclusive entre profissionais de um mesmo serviço. (Tabela 1) 
Tabela 1

Freqüência absoluta e relativa dos profissionais de saúde que responderam o questionário, referente a algumas das questões propostas.

\begin{tabular}{|c|c|c|c|}
\hline & & $\mathbf{n}$ & $\%$ \\
\hline \multirow[t]{5}{*}{ Número de lâmpadas por aparelho de fototerapia } & $<6$ & 9 & 10,0 \\
\hline & 6 & 57 & 65,0 \\
\hline & 7 & 18 & 20,0 \\
\hline & 8 & 5 & 5,0 \\
\hline & Total & 89 & 100,0 \\
\hline \multirow[t]{9}{*}{ Período de checagem da irradiância (dias) } & Diária & 2 & 4,0 \\
\hline & 2 & 5 & 11,0 \\
\hline & 7 & 2 & 4,0 \\
\hline & 15 & 6 & 14,0 \\
\hline & 21 & 1 & 2,0 \\
\hline & 30 & 5 & 11,0 \\
\hline & 60 & 3 & 7,0 \\
\hline & NS & 21 & 47,0 \\
\hline & Total & 45 & 100,0 \\
\hline \multirow[t]{7}{*}{ Distância fonte luminosa - RN (cm) } & 20 & 2 & 3,0 \\
\hline & 30 & 20 & 22,0 \\
\hline & 40 & 17 & 20,0 \\
\hline & 50 & 27 & 31,0 \\
\hline & 60 & 9 & 10,0 \\
\hline & 70 & 12 & 14,0 \\
\hline & Total & 87 & 100,0 \\
\hline \multirow[t]{5}{*}{ Volume de aumento da taxa hídrica ( $\mathrm{ml} / \mathrm{kg})$} & 10 & 15 & 47,0 \\
\hline & 20 & 13 & 40,0 \\
\hline & 40 & 1 & 3,0 \\
\hline & NS & 5 & 10,0 \\
\hline & Total & 32 & 100,0 \\
\hline
\end{tabular}

\section{Cuidados gerais com o RN em fototerapia}

Cem por cento dos médicos prescrevem a proteção ocular para o RN durante a fototerapia, sendo que a proteção das gônadas foi recomendada por apenas $5 \%$ dos profissionais.

Embora a maioria dos profissionais médicos e de enfermagem (61\%) não recomende a utilização de fraldas em recém-nascidos submetidos à fototerapia, $39 \%$ deles recomendam o seu uso rotineiro.

Todos os profissionais de saúde entrevistados são a favor da manutenção do aleitamento materno para recém-nascidos submetidos à fototerapia.

Dos 89 profissionais entrevistados, 82 (92\%) afirmaram que a mudança de decúbito é realizada regularmente em pacientes recebendo fototerapaia.
Entretanto, quando solicitados a precisar a frequiência de mudança, observamos grande variabilidade nas respostas, inclusive entre profissionais de um mesmo serviço, cujas respostas variavam de 2 a 12 mudanças de decúbito por dia.

A prescrição, quando necessária, de fototerapia dupla (utilização de dois aparelhos de fototerapia em uma mesma criança) foi confirmada por todos os médicos entrevistados.

Quando são utilizados os spots halógenos, 30\% dos médicos recomendaram que os focos luminosos fossem concêntricos entre si, $30 \%$ recomendaram focos luminosos tangenciais e $40 \%$ não recomendaram direcionamento específico dos focos, mas sim, que fossem direcionados aleatoriamente a qualquer parte do corpo do recém-nascido. 
Comparação entre as respostas dos médicos entrevistados quanto aos níveis séricos de bilirrubina (Bb) indicativos de fototerapia e exanguineotransfusão (EXT) para RNs com peso de nascimento de $1000 \mathrm{~g}, 1500 \mathrm{~g}$ e $2500 \mathrm{~g}$ e as indicações da Academia Americana de Pediatria (AAP).

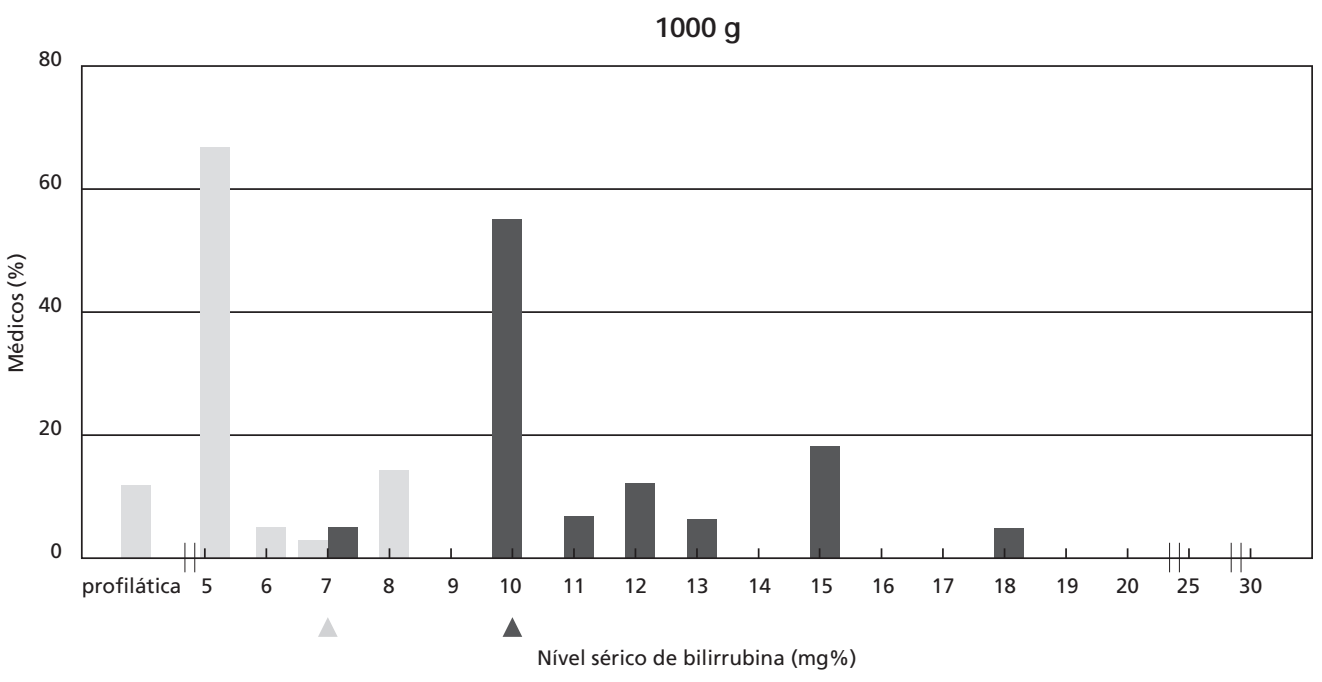

Fototerapia

Exsanguineotransfusão

Nível sérico de $\mathrm{Bb}$ indicativo de fototerapia pela APP

- Nível sérico de $\mathrm{Bb}$ indicativo de EXT pela APP

\section{$1500 \mathrm{~g}$}

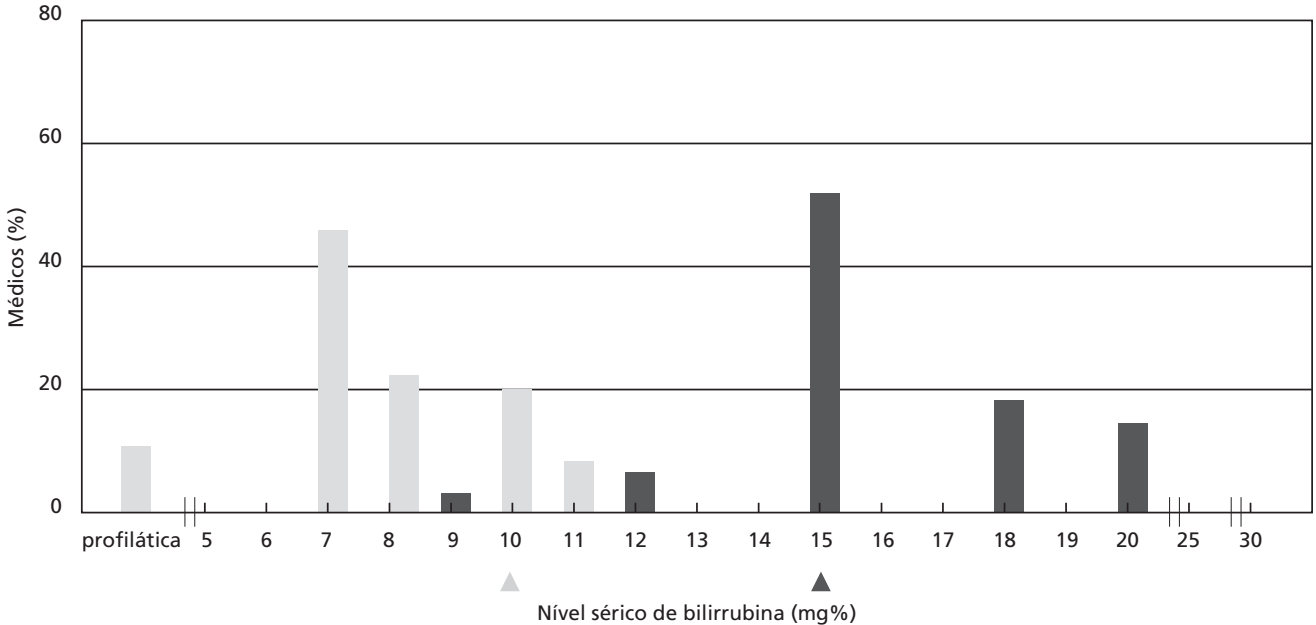

Fototerapia

Exsanguineotransfusão

Nível sérico de $\mathrm{Bb}$ indicativo de fototerapia pela APP

Nível sérico de $\mathrm{Bb}$ indicativo de EXT pela APP

\section{$2500 \mathrm{~g}$}

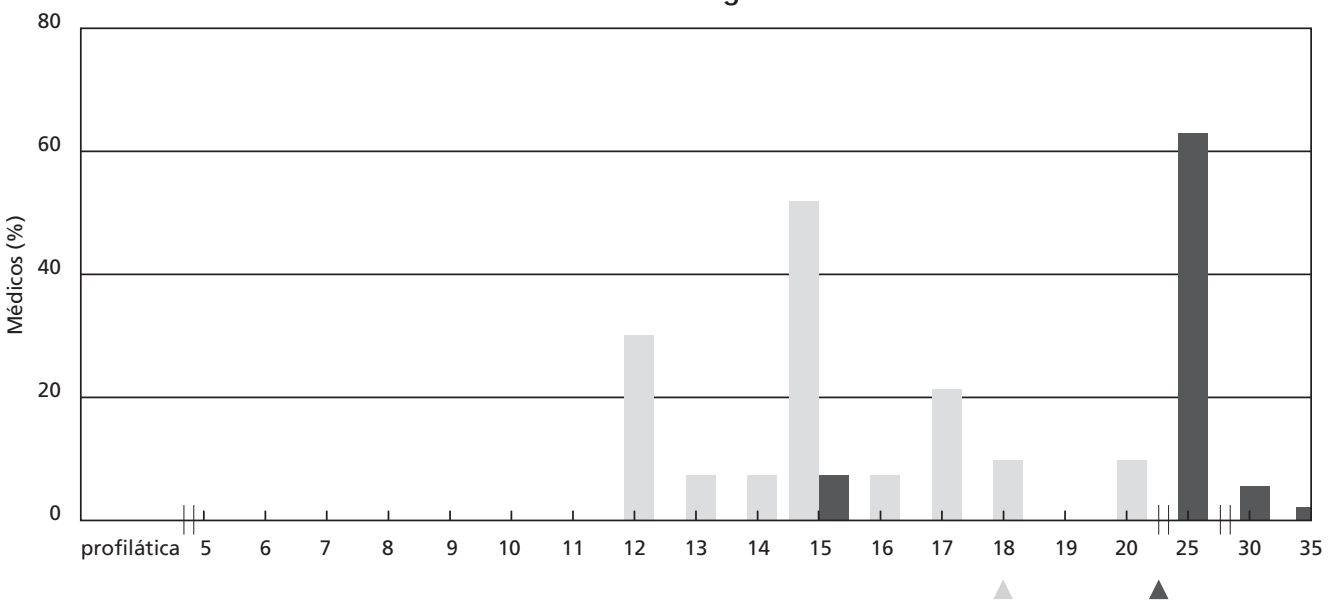

Fototerapia

Exsanguineotransfusão

Nível sérico de $\mathrm{Bb}$ indicativo de fototerapia pela APP

Nível sérico de $\mathrm{Bb}$ indicativo de EXT pela APP 
Níveis séricos de bilirrubina indicativos de tratamento

Cinqüenta e oito por cento dos médicos entrevistados afirmaram que determinam o nível sérico de bilirrubina em recém-nascidos submetidos à fototerapia uma vez ao dia, $14 \%$ duas vezes ao dia e os $28 \%$ restantes não souberam precisar a frequiência.

Setenta e quatro por cento dos médicos afirmaram prescrever fototerapia profilática para os recém-nascidos prematuros, principalmente quando o seu peso ao nascimento era inferior à $1500 \mathrm{~g}$ e/ou apresentava equimoses.

Todos os médicos afirmaram existir uma tabela contendo os níveis séricos de bilirrubina indicativos de fototerapia em suas unidades. Entretanto, em relação à tabela contendo os níveis séricos de bilirrubina indicativos de EXT, quarenta e sete médicos afirmaram sua existência, dois afirmaram que ela não existia e um médico não soube informar.

Apesar do relato da existência dessa tabela observamos uma grande variabilidade nas respostas dadas pelos médicos quanto ao início do tratamento em RN ictéricos em três diferentes faixas de peso (1000 g, 1500 g e 2500 g). Por exemplo, para um recém-nascido pesando $1500 \mathrm{~g}, 10 \%$ dos médicos afirmaram que recomendariam fototerapia profilática, $46 \%$ afirmaram que iniciariam fototerapia com níveis séricos de bilirrubina maior que $7 \mathrm{mg} \%, 36 \%$ indicariam tal tratamento com níveis maiores que 8 $\mathrm{mg} \%$ e $8 \%$ dos profissionais iniciariam fototerapia quando os níveis séricos de bilirrubina atingissem níveis iguais ou superiores a $11 \mathrm{mg} \%$. (Figura 1)

O mesmo quadro de variabilidade nas respostas foi também observado quanto ao nível sérico de bilirrubina indicativo de tratamento fototerápico ou EXT em recém-nascidos com peso ao nascimento de 1000 g e 2500 g. (Figura 1)

\section{Discussão}

A icterícia é a patologia mais freqüente no período neonatal. Apesar da alta incidência, sua abordagem clínica continua cercada de controvérsias. 6

$\mathrm{O}$ ato de expor à luz, ao iniciar a fototerapia, não implica, necessariamente, que o RN esteja recebendo tratamento adequado. Fatores que, inicialmente, parecem ter pouca relevância podem interferir de forma determinante na eficácia do tratamento. ${ }^{7}$

Os resultados de nosso estudo sugerem que algumas práticas adotadas pelos profissionais de saúde podem diminuir a eficácia da fototerapia.

\section{Intensidade luminosa e superfície corporal exposta à luz}

Como a luz interage com o pigmento bilirrubínico depositado na pele do $\mathrm{RN}$, quanto maior a superfície corporal exposta à luz, maior será a eficácia da fototerapia. ${ }^{8,9}$ Dessa forma, o uso de fraldas é contraindicado e esse aspecto é freqüentemente negligenciado, como sugere o resultado da pesquisa, na qual $39 \%$ dos entrevistados recomendaram o uso rotineiro de fraldas durante a fototerapia.

No mesmo contexto, em RN com peso superior a $2500 \mathrm{~g}$ e que esteja recebendo fototerapia com emissão de luz em foco (Bilispot®), a eficácia terapêutica pode ser consideravelmente aumentada com a inclusão de focos adicionais (fototerapia dupla ou tripla). ${ }^{8}$ Esses focos não devem ser direcionados concentricamente mas de maneira que iluminem a maior superfície corporal possível (focos tangenciais). Em nosso estudo, $30 \%$ dos profissionais entrevistados recomendaram que os focos luminosos fossem direcionados de forma concêntrica e $40 \%$ de forma aleatória.

A utilização de fototerapia profilática em RN menor que $1500 \mathrm{~g}$ e com equimoses, indicada por $74 \%$ dos médicos entrevistados, e a utilização de proteção das gônadas contra a luz, relatada por 5\% dos médicos, são achados que não encontram base na literatura médica atual.4,6

Outro fator importante para a eficácia da fototerapia é a irradiância na pele do RN, sendo inversamente proporcional à distância entre a fonte luminosa e o paciente. ${ }^{9}$ Quando um aparelho de fototerapia convencional equipado com sete lâmpadas fluorescentes brancas (tipo luz do dia) é posicionado a $50 \mathrm{~cm}$ de um RN, a irradiância medida na superfície corporal é de $4 \mu \mathrm{w} / \mathrm{cm}^{2} / \mathrm{nm}$ e quando posicionado a $20 \mathrm{~cm}$, aumenta para $8 \mu \mathrm{w} / \mathrm{cm}^{2} / \mathrm{nm}$. A cada centímetro que a fonte luminosa é aproximada do paciente, um incremento de $3 \%$ na irradiância é observado.

Tendo por base esse conhecimento, estima-se que a distância mais apropriada entre o $\mathrm{RN}$ e a fonte luminosa seja de $30 \mathrm{~cm}$, já que distâncias menores que essa, apesar de aumentarem a irradiância, aumentam o risco de hipertermia. ${ }^{10}$ Embora 96\% dos profissionais entrevistados tenham afirmado a existência de uma distância padronizada para o posicionamento dos aparelhos de fototerapia em relação ao RN, observamos enorme discrepância nas respostas fornecidas, que variaram de 20 a $70 \mathrm{~cm}$.

Para assegurar a eficácia da fototerapia é importante que a aferição da irradiância seja regular, para 
garantir que o paciente receba, pelo menos, a "dose mínima eficaz" ( $\left.4 \mu \mathrm{w} / \mathrm{cm}^{2} / \mathrm{nm}\right) .11$ Embora $50 \%$ dos profissionais entrevistados tenham afirmado existir uma rotina para essa checagem, a maioria não soube informar sua freqüência, de que maneira ou com que instrumento ela era realizada. Em um mesmo serviço, alguns profissionais relataram que a aferição da irradiância era realizada diariamente, enquanto outros afirmaram que ocorria a cada 60 dias.

Com o uso, lâmpadas fluorescentes tendem a emitir menor intensidade luminosa e devem ser trocadas quando a irradiância emitida for menor que a "dose mínima eficaz". ${ }^{12}$ Trinta e quatro por cento dos profissionais entrevistados declararam que as lâmpadas fluorescentes eram trocadas regularmente, embora não soubessem precisar a freqüência, $37 \%$ afirmaram que elas nunca eram trocadas e $29 \%$ não souberam responder tal questionamento.

\section{Cuidados gerais com o RN}

durante o uso da fototerapia

Houve concordância entre os entrevistados em relação ao incentivo e a manutenção do aleitamento materno para $\mathrm{RN}$ ictérico durante a fototerapia.

Grande controvérsia, porém, foi verificada no tocante ao aumento da oferta hídrica ao RN durante a fototerapia, afirmada por $64 \%$ dos médicos. Esse aumento da oferta de líquidos variou de 5 a 40 $\mathrm{ml} / \mathrm{kg} / \mathrm{dia}$, inclusive entre profissionais de um mesmo serviço.

Na literatura médica, entretanto, não há recomendação específica e objetiva sobre isto. ${ }^{6}$ Embora ocorra maior perda de água corporal durante a fototerapia, principalmente nos RNs prematuros e de muito baixo peso, o aumento da oferta hídrica deve ser avaliado de acordo com as particularidades de cada caso. A AAP não recomenda a instituição de hidratação venosa ou hiper-hidratação nos RN ictéricos em fototerapia. Recomenda, sim, para o RN desidratado, o aumento da oferta de líquidos, preferencialmente pela via entérica, com uso de leite materno ou fórmulas lácteas apropriadas, já que, além de promover a reidratação, diminui a circulação êntero-hepática e aumenta a excreção dos metabólitos da bilirrubina. ${ }^{4}$

Embora não haja evidência científica de que a mudança regular de decúbito em $\mathrm{RN}$ recebendo fototerapia aumente a eficácia terapêutica, $92 \%$ dos profissionais afirmaram adotar (esta) essa prática, variando a freqüência de mudanças de 2 a 12 por dia. 10

\section{Níveis séricos de bilirrubina indicativos de fototerapia e exanguineotransfusão}

Apesar do relato da existência de tabelas que norteiam a decisão da prescrição de fototerapia e/ou exanguineotranfusão, baseados nos níveis séricos de bilirrubina e no peso de nascimento, nossos resultados demonstram enorme variação nas indicações dessas modalidades terapêuticas. A variabilidade era tanto maior quanto menor fosse o peso do RN.

Por exemplo, para um RN com peso de nascimento de $1500 \mathrm{~g}$, os níveis séricos de bilirrubina indicativos de fototerapia variaram, segundo as respostas dos médicos, de menos de $5 \mathrm{mg} \%$ a $11 \mathrm{mg} \%$. Para indicar exanguineotransfusão, esses valores variaram de 12 a $20 \mathrm{mg} \%$.

$\mathrm{O}$ fato de profissionais seguirem condutas tão diversas em relação às questões básicas para a eficácia do tratamento fototerápico permite colocar em dúvida não só a aplicabilidade das normas de condutas, como também, e principalmente, a falta de adesão a uma prática baseada em evidências científicas. 13,14

Paira, em decorrência, a dúvida do real conhecimento dessas normas de conduta pelos profissionais que devem utilizá-las e da discussão correta das mesmas previamente à sua inserção em cada unidade.

Cabana et al.,15 em estudo publicado em 1999, relatam uma série de motivos que interferem na aderência às normas de condutas pelos profissionais de saúde, onde destacamos a falta de familiaridade com as novas regras, resistência em alterar práticas antigas, desconhecimento dos desfechos clínicos conseqüentes do não cumprimento das normas, a discordância e o descrédito na eficiência das práticas sugeridas.

Uma recente pesquisa avaliou a aplicação das normas recomendadas pela AAP para a instituição de fototerapia e exanguineotransfusão em RN a termo e saudável em hospitais norte-americanos e discutiu os motivos da dificuldade de aderência às normas de conduta em geral, revelando, no caso específico, uma aderência de apenas $66 \% .16$

\section{Conclusões}

Todas as unidades pesquisadas dispõem de aparelhos de fototerapia e a maioria delas alega possuir uma norma de conduta para seu próprio uso. Grandes discrepâncias, entretanto, foram encontradas em sua utilização, principalmente entre profissionais de uma mesma unidade, teoricamente seguindo a mesma norma de conduta. 
Observa-se, também, que além dos entrevistados desconhecerem rotinas pré-estabelecidas em suas unidades e não verificarem a qualidade do tratamento fototerápico oferecido, adotam, muitas vezes, condutas que certamente reduzem a eficácia do tratamento.

O uso inadequado dessa tecnologia expõe o RN a exames laboratoriais desnecessários, prolonga seu período de hospitalização e interfere na relação mãebebê.

Todos os profissionais de saúde que utilizam a fototerapia precisam estar informados das normas e rotinas existentes, conscientizados da necessidade de sua aderência a elas, treinados adequadamente para aplicá-las, além de avaliar regularmente a eficácia dessa modalidade terapêutica.

\section{Referências}

1. DeCarvalho M. Hiperbilirrubinemia neonatal. Temas Pediatr 1989, 45: 1-22.

2. DeCarvalho M, Lopes JM. Qual o tempo de vida útil de lâmpadas fluorescentes para fototerapia? J Pediatr (Rio J) 1991; 67: 151-6.

3. Bratlid D. Criteria for treatment of neonatal jaundice. J Perinatol 2001; 21 [Suppl]1: S88-92.

4. AAP (American Academy of Pediatrics). Practice parameter: management of hyperbilirrubinemia in the healthy term newborn. Pediatrics 1994; 94: 558-62.

5. Hansen TWR. Therapeutic approaches to neonatal jaundice: a international survey. Clin Pediatr 1996; 35: 309-16.

6. Fanaroff AA, Martin RJ. Neonatal jaundice and liver disease. In: Young LD, editor. Neonatal-perinatal medicine. Saint Louis: Year Book; 1997. p.1345-89.

7. DeCarvalho M, Lopes JM. Fototerapia nos hospitais públicos do Rio de Janeiro. J Pediatr (Rio J) 1991; 67: 15762

8. Tan KL. The nature of the dose-response relationship of phototherapy for neonatal hyperbilirrubinemia. J Pediatr 1997; 90: 448-52.

9. Maisels MJ. Why use homeopathic doses of phototherapy? Pediatrics 1996; 98: 283-7.
10. DeCarvalho M. Fototerapia de alta intensidade para o tratamento da hiperbilirrubinemia neonatal: estudo da eficácia terapêutica [tese doutorado]. Rio de Janeiro: Pósgraduação em Saúde da Criança e da Mulher/IFF/ FIOCRUZ; 1998.

11. Hansen TWR. Acute management of extreme neonatal jaundice - the potencial benefits of intensified phototherapy and interruption of entherohepatic bilirrubin circulation. Clin Pediatr. 1997, 86: 843-6.

12. Canadian Pediatric Society, Fetus and Newborn Commitee. Approach to the management of bilirrubinemia in term newborn infants. Paediatr Child Health 1999; 4: 161-4.

13. Bergman DA. Evidence-based guidelines and critical pathways for quality improvement. Pediatrics 1987; 103 : 225-32.

14. AAP (American Academy of Pediatrics). Guiding principles, attributes and process to review medical management guidelines. Pediatrics 2001; 108: 1378-82.

15. Cabana MD, Rand CS, Powe NR, Wu AW, Wilson MH, Abboud PA, Rubin HR. Why don't physicians follow clinical practice guidelines? JAMA 1999; 282: 1458-65.

16. Atikinson LR, Escobar GJ, Takayama JI, Newman TB. Phototherapy use in jaundice newborns in a large managed care organization: do clinicians adhere to the guideline? Pediatrics 2003; 111: 555-61.

Recebido em 2 de julho de 2004

Versão final apresentada em 29 de outubro de 2004

Aprovado em 10 de novembro de 2004 